\title{
Decoupling the Stationary Navier-Stokes-Darcy System with the Beavers-Joseph-Saffman Interface Condition
}

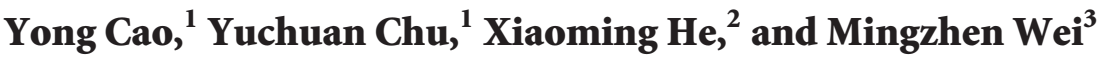 \\ ${ }^{1}$ Department of Mechanical Engineering \& Automation, Harbin Institute of Technology, Shenzhen Graduate School, \\ Shenzhen, Guangdong 518055, China \\ ${ }^{2}$ Department of Mathematics and Statistics, Missouri University of Science and Technology, Rolla, MO 65409, USA \\ ${ }^{3}$ Department of Geological Science and Engineering, Missouri University of Science and Technology, Rolla, MO 65409, USA
}

Correspondence should be addressed to Xiaoming He; hex@mst.edu

Received 5 April 2013; Accepted 31 July 2013

Academic Editor: R. K. Bera

Copyright (C) 2013 Yong Cao et al. This is an open access article distributed under the Creative Commons Attribution License, which permits unrestricted use, distribution, and reproduction in any medium, provided the original work is properly cited.

This paper proposes a domain decomposition method for the coupled stationary Navier-Stokes and Darcy equations with the Beavers-Joseph-Saffman interface condition in order to improve the efficiency of the finite element method. The physical interface conditions are directly utilized to construct the boundary conditions on the interface and then decouple the Navier-Stokes and Darcy equations. Newton iteration will be used to deal with the nonlinear systems. Numerical results are presented to illustrate the features of the proposed method.

\section{Introduction}

The Stokes-Darcy model has been extensively studied in the recent years due to its wide range of applications in many natural world problems and industrial settings, such as the subsurface flow in karst aquifers, oil flow in vuggy porous media, industrial filtrations, and the interaction between surface and subsurface flows [1-8]. Since the problem domain naturally consists of two different physical subdomains, several different numerical methods have been developed to decouple the Stokes and Darcy equations [6, 9-26]. For other works on the numerical methods and analysis of the StokesDarcy model, we refer the readers to [27-45].

Recently the more physically valid Navier-Stokes-Darcy model has attracted scientists' attention, and several coupled finite element methods have been studied for it [46-51]. On the other hand, the advantages of the domain decomposition methods (DDMs) in parallel computation and natural preconditioning have motivated the development of different DDMs for solving the Stoke-Darcy model [6, 10-18, 21, 22]. In this paper, we will develop a domain decomposition method for the Navier-Stokes-Darcy model based on Robin boundary conditions constructed from the interface conditions. This physics-based DDM is different from the traditional ones in the sense that they focus on decomposing different physical domains by directly utilizing the given physical interface conditions.

The rest of paper is organized as follows. In Section 2, we introduce the Navier-Stokes-Darcy model with the BeaversJoseph-Saffman interface condition. In Section 3, we recall the coupled weak formulation and the corresponding coupled finite element method for the Navier-Stokes-Darcy model. In Section 4, a parallel domain decomposition method and its finite element discretization are proposed to decouple the Navier-Stokes-Darcy system by using the Robin-type boundary conditions constructed from the physical interface conditions. Finally, in Section 5, we present a numerical example to illustrate the features of the proposed method.

\section{Stationary Navier-Stokes-Darcy Model}

In this section we introduce the following coupled NavierStokes-Darcy model on a bounded domain $\Omega=\Omega_{m} \cup \Omega_{c} \subset$ $\mathbb{R}^{d},(d=2,3)$; see Figure 1 . In the porous media region $\Omega_{m}$, the flow is governed by the Darcy system

$$
\begin{gathered}
\vec{u}_{m}=-\mathbb{K} \nabla \phi_{m}, \\
\nabla \cdot \vec{u}_{m}=f_{m} .
\end{gathered}
$$




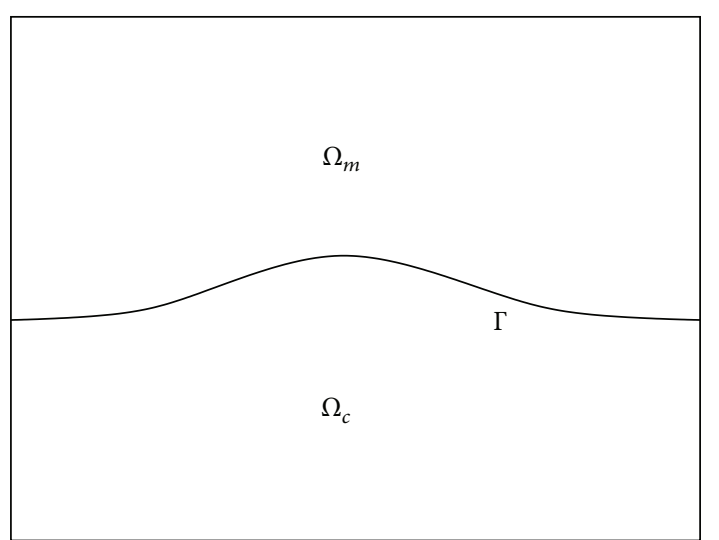

FIGURE 1: A sketch of the porous median domain $\Omega_{m}$, fluid domain $\Omega_{c}$, and the interface $\Gamma$.

Here, $\vec{u}_{m}$ is the fluid discharge rate in the porous media, $\mathbb{K}$ is the hydraulic conductivity tensor, $f_{m}$ is a sink/source term, and $\phi_{m}$ is the hydraulic head defined as $z+\left(p_{m} / \rho g\right)$, where $p_{m}$ denotes the dynamic pressure, $z$ the height, $\rho$ the density, and $g$ the gravitational acceleration. We will consider the following second-order formulation, which eliminates $\vec{u}_{m}$ in the Darcy system:

$$
-\nabla \cdot\left(\mathbb{K} \nabla \phi_{m}\right)=f_{m}
$$

In the fluid region $\Omega_{c}$, the fluid flow is assumed to be governed by the Navier-Stokes equations:

$$
\begin{gathered}
\vec{u}_{c} \cdot \nabla \vec{u}_{c}-\nabla \cdot \mathbb{T}\left(\vec{u}_{c}, p_{c}\right)=\vec{f}_{c}, \\
\nabla \cdot \vec{u}_{c}=0,
\end{gathered}
$$

where $\vec{u}_{c}$ is the fluid velocity, $p_{c}$ is the kinematic pressure, $\vec{f}_{c}$ is the external body force, $v$ is the kinematic viscosity of the fluid, $\mathbb{T}\left(\vec{u}_{c}, p_{c}\right)=2 \nu \mathbb{D}\left(\vec{u}_{c}\right)-p_{c} \rrbracket$ is the stress tensor, and $\mathbb{D}\left(\vec{u}_{c}\right)=\left(\nabla \vec{u}_{c}+\nabla^{T} \vec{u}_{c}\right) / 2$ is the deformation tensor.

Let $\Gamma=\bar{\Omega}_{m} \cap \bar{\Omega}_{c}$ denote the interface between the fluid and porous media regions. On the interface $\Gamma$, we impose the following three interface conditions:

$$
\begin{gathered}
\vec{u}_{c} \cdot \vec{u}_{c}=-\vec{u}_{m} \cdot \vec{n}_{m}, \\
-\vec{u}_{c} \cdot\left(\mathbb{T}\left(\vec{u}_{c}, p_{c}\right) \cdot \vec{n}_{c}\right)=g\left(\phi_{m}-z\right), \\
-\boldsymbol{\tau}_{j} \cdot\left(\mathbb{T}\left(\vec{u}_{c}, p_{c}\right) \cdot \vec{n}_{c}\right)=\frac{\alpha \nu \sqrt{\mathbf{d}}}{\sqrt{\operatorname{trace}\left(\prod\right)}} \boldsymbol{\tau}_{j} \cdot \vec{u}_{c},
\end{gathered}
$$

where $\vec{n}_{c}$ and $\vec{n}_{m}$ denote the unit outer normal to the fluid and the porous media regions at the interface $\Gamma$, respectively, $\boldsymbol{\tau}_{j}(j=1, \ldots, d-1)$ denote mutually orthogonal unit tangential vectors to the interface $\Gamma$, and $\Pi=\mathbb{K} v / g$. The third condition (7) is referred to as the Beavers-Joseph-Saffman (BJS) interface condition [52-55].

In this paper, for simplification, we assume that the hydraulic head $\phi_{m}$ and the fluid velocity $\vec{u}_{c}$ satisfy the homogeneous Dirichlet boundary condition except on $\Gamma$, that is, $\phi_{m}=0$ on the boundary $\partial \Omega_{m} / \Gamma$ and $\vec{u}_{c}=0$ on the boundary $\partial \Omega_{c} / \Gamma$.

\section{Coupled Weak Formulation and Finite Element Method}

In this section we will recall the coupled weak formulation and the corresponding coupled finite element method for the Navier-Stokes-Darcy model with Beavers-Joseph-Saffman condition. Let $(\cdot, \cdot)_{D}$ denote the $L^{2}$ inner product on the domain $D\left(D=\Omega_{c}\right.$ or $\left.\Omega_{m}\right)$ and let $\langle\cdot, \cdot\rangle$ denote the $L^{2}$ inner product on the interface $\Gamma$ or the duality pairing between $\left(H_{00}^{1 / 2}(\Gamma)\right)^{\prime}$ and $H_{00}^{1 / 2}(\Gamma)$ [5]. Define the spaces

$$
\begin{gathered}
X_{c}=\left\{\vec{v} \in\left[H^{1}\left(\Omega_{c}\right)\right]^{d} \mid \vec{v}=0 \text { on } \frac{\partial \Omega_{c}}{\Gamma}\right\}, \\
Q_{c}=L^{2}\left(\Omega_{c}\right), \\
X_{m}=\left\{\psi \in H^{1}\left(\Omega_{m}\right) \mid \psi=0 \text { on } \frac{\partial \Omega_{m}}{\Gamma}\right\},
\end{gathered}
$$

the bilinear forms

$$
\begin{gathered}
a_{m}\left(\phi_{m}, \psi\right)=\left(\mathbb{K} \nabla \phi_{m}, \nabla \psi\right)_{\Omega_{m}}, \\
a_{c}\left(\vec{u}_{c}, \vec{v}\right)=2 v\left(\mathbb{D}\left(\vec{u}_{c}\right), \mathbb{D}(\vec{v})\right)_{\Omega_{c}}, \\
b_{c}(\vec{v}, q)=-(\nabla \cdot \vec{v}, q)_{\Omega_{c}},
\end{gathered}
$$

and the projection onto the tangent space on $\Gamma$ :

$$
P_{\tau} \vec{u}=\sum_{j=1}^{d-1}\left(\vec{u} \cdot \boldsymbol{\tau}_{j}\right) \boldsymbol{\tau}_{j} .
$$

With these notations, the weak formulation of the coupled Navier-Stokes-Darcy model with BJS interface condition is given as follows [46-51]: find $\left(\vec{u}_{c}, p_{c}, \phi_{m}\right) \in X_{c} \times Q_{c} \times X_{m}$ such that

$$
\begin{aligned}
\left(\vec{u}_{c} \cdot\right. & \left.\nabla \vec{u}_{c}, \vec{v}\right)_{\Omega_{c}}+a_{c}\left(\vec{u}_{c}, \vec{v}\right)+b_{c}\left(\vec{v}, p_{c}\right) \\
& -b_{c}\left(\vec{u}_{c}, q\right)+a_{m}\left(\phi_{m}, \psi\right) \\
& +\left\langle g \phi_{m}, \vec{v} \cdot \vec{n}_{c}\right\rangle-\left\langle\vec{u}_{c} \cdot \vec{n}_{c}, \psi\right\rangle \\
& +\frac{\alpha \nu \sqrt{\mathbf{d}}}{\sqrt{\operatorname{trace}\left(\prod\right)}}\left\langle P_{\tau} \vec{u}_{c}, P_{\tau} \vec{v}\right\rangle \\
= & \left(f_{m}, \psi\right)_{\Omega_{m}}+\left(\vec{f}_{c}, \vec{v}\right)_{\Omega_{c}} \\
& +\left\langle g z, \vec{v} \cdot \vec{n}_{c}\right\rangle, \quad \forall(\vec{v}, q, \psi) \in X_{c} \times Q_{c} \times X_{m} .
\end{aligned}
$$

Assume that we have in hand regular subdivisions of $\Omega_{m}$ and $\Omega_{c}$ into finite elements with mesh size $h$. Then one can define finite element spaces $X_{m h} \subset X_{m}, X_{c h} \subset X_{c}$ and 
$Q_{c h} \subset Q_{c}$. We assume that $X_{c h}$ and $Q_{c h}$ satisfy the inf-sup condition $[56,57]$

$$
\inf _{0 \neq q_{h} \in Q_{c h}} \sup _{0 \neq \vec{v}_{h} \in X_{c h}} \frac{b_{c}\left(\vec{v}_{h}, q_{h}\right)}{\left\|\vec{v}_{h}\right\|_{1}\left\|q_{h}\right\|_{0}}>\gamma,
$$

where $\gamma>0$ is a constant independent of $h$. This condition is needed in order to ensure that the spatial discretizations of the Navier-Stokes equations used here are stable. See $[56,57]$ for more details of finite element spaces $X_{m h}, X_{c h}$, and $Q_{c h}$ that satisfy (12). One example is the Taylor-Hood element pair that we use in the numerical experiments; for that pair, $X_{c h}$ consists of continuous piecewise quadratic polynomials and $Q_{c h}$ consists of continuous piecewise linear polynomials.

Then a coupled finite element method with Newton iteration for the coupled Navier-Stokes-Darcy model is given as follows [46]: find $\left(\vec{u}_{c, h}, p_{c, h}, \phi_{m, h}\right) \in X_{c h} \times Q_{c h} \times X_{m h}$ in the following procedure.

(1) The initial value $\vec{u}_{c, h}^{0}$ is chosen.

(2) For $m=0,1,2, \ldots, M$, solve

$$
\begin{aligned}
\left(\vec{u}_{c, h}^{m+1}\right. & \left.\cdot \nabla \vec{u}_{c, h}^{m}, \vec{v}_{h}\right)_{\Omega_{c}}+\left(\vec{u}_{c, h}^{m} \cdot \nabla \vec{u}_{c, h}^{m+1}, \vec{v}_{h}\right)_{\Omega_{c}} \\
& +a_{c}\left(\vec{u}_{c, h}^{m+1}, \vec{v}_{h}\right)+b_{c}\left(\vec{v}_{h}, p_{c, h}^{m+1}\right) \\
& -b_{c}\left(\vec{u}_{c, h}^{m+1}, q_{h}\right)+a_{m}\left(\phi_{m, h}^{m+1}, \psi_{h}\right) \\
& +\left\langle g \phi_{m, h}^{m+1}, \vec{v}_{h} \cdot \vec{n}_{c}\right\rangle-\left\langle\vec{u}_{c, h}^{m+1} \cdot \vec{n}_{c}, \psi_{h}\right\rangle \\
& +\frac{\alpha \nu \sqrt{\mathbf{d}}}{\sqrt{\operatorname{trace}\left(\prod\right)}}\left\langle P_{\tau} \vec{u}_{c, h}^{m+1}, P_{\tau} \vec{v}_{h}\right\rangle \\
= & \left(\vec{u}_{c, h}^{m} \cdot \nabla \vec{u}_{c, h}^{m}, \vec{v}_{h}\right)_{\Omega_{c}}+\left(f_{m}, \psi_{h}\right)_{\Omega_{m}} \\
& +\left(\vec{f}_{c}, \vec{v}_{h}\right)_{\Omega_{c}}+\left\langle g z, \vec{v}_{h} \cdot \vec{n}_{c}\right\rangle, \\
\forall & \left(\vec{v}_{h}, q_{h}, \psi_{h}\right) \in X_{c h} \times Q_{c h} \times X_{m h} .
\end{aligned}
$$

(3) Set $\vec{u}_{c, h}=\vec{u}_{c, h}^{m+1}, p_{c, h}=\vec{p}_{c, h}^{m+1}$, and $\phi_{m, h}=\phi_{m, h}^{M+1}$.

\section{Physics-Based Domain Decomposition Method}

The coupled finite element method may end up with a huge algebraic system, which combines all parts from the Navier-Stokes equations, Darcy equation, and interface conditions together into one sparse matrix. Hence it is often impractical to directly apply this method to large-scale real world applications. In order to develop a more efficient numerical method in this section, we will directly utilize the three physical interface conditions to construct a physicsbased parallel domain decomposition method to decouple the Navier-Stokes and Darcy equations.
Let us first consider the following Robin condition for the Darcy system: for a given constant $\gamma_{p}>0$ and a given function $\eta_{p}$ defined on $\Gamma$,

$$
\gamma_{p} \mathbb{K} \nabla \widehat{\phi}_{m} \cdot \vec{n}_{m}+g \widehat{\phi}_{m}=\eta_{p}, \quad \text { on } \Gamma \text {. }
$$

Then, the corresponding weak formulation for the Darcy part is given by the following: for $\eta_{p} \in L^{2}(\Gamma)$, find $\widehat{\phi}_{m} \in X_{m}$ such that

$$
\begin{aligned}
& a_{m}\left(\widehat{\phi}_{m}, \psi\right)+\left\langle\frac{g \widehat{\phi}_{m}}{\gamma_{p}}, \psi\right\rangle \\
& \quad=\left(f_{m}, \psi\right)_{\Omega_{m}}+\left\langle\frac{\eta_{p}}{\gamma_{p}}, \psi\right\rangle, \quad \forall \psi \in X_{m} .
\end{aligned}
$$

Second, we can propose the following two Robin-type conditions for the Navier-Stokes equations: for a given constant $\gamma_{f}>0$ and given functions $\eta_{f}$ and $\vec{\eta}_{f \tau}$ defined on $\Gamma$,

$$
\begin{gathered}
\vec{n}_{c} \cdot\left(\mathbb{T}\left(\widehat{\vec{u}}_{c}, \widehat{p}_{c}\right) \cdot \vec{n}_{c}\right)+\gamma_{f} \widehat{\vec{u}}_{c} \cdot \vec{n}_{c}=\eta_{f}, \quad \text { on } \Gamma, \\
-P_{\tau}\left(\mathbb{T}\left(\widehat{\vec{u}}_{c}, p_{c}\right) \cdot \vec{n}_{c}\right)=\frac{\alpha \nu \sqrt{\mathbf{d}}}{\sqrt{\operatorname{trace}\left(\prod\right)}} P_{\tau} \widehat{\vec{u}}_{c}, \quad \text { on } \Gamma .
\end{gathered}
$$

Then, the corresponding weak formulation for the Navier-Stokes equation is given by the following: for $\eta_{f} \in$ $L^{2}(\Gamma)$, find $\left(\widehat{\vec{u}}_{c}, \widehat{p}_{c}\right) \in X_{c} \times Q_{c}$ such that

$$
\begin{aligned}
\left(\widehat{\vec{u}}_{c} \cdot \nabla \widehat{\vec{u}}_{c}, \vec{v}\right)_{\Omega_{c}}+a_{c}\left(\widehat{\vec{u}}_{c}, \vec{v}\right)+b_{c}\left(\vec{v}, \widehat{p}_{c}\right) \\
\quad-b_{c}\left(\widehat{\vec{u}}_{c}, q\right)+\gamma_{f}\left\langle\widehat{\vec{u}}_{c} \cdot \vec{n}_{c}, \vec{v} \cdot \vec{u}_{c}\right\rangle \\
\quad+\frac{\alpha \nu \sqrt{\mathbf{d}}}{\sqrt{\operatorname{trace}\left(\prod\right)}}\left\langle P_{\tau} \widehat{\vec{u}}_{c}, P_{\tau} \vec{v}\right\rangle \\
=\left(\vec{f}_{c}, \vec{v}\right)_{\Omega_{c}}+\left\langle\eta_{f}, \vec{v} \cdot \vec{n}_{c}\right\rangle, \quad \forall(\vec{v}, q) \in X_{c} \times Q_{c} .
\end{aligned}
$$

Our next step is to show that, for appropriate choices of $\gamma_{f}, \gamma_{\mathrm{p}}, \eta_{f}$, and $\eta_{p}$, the solutions of the coupled system (11) are equivalent to the solutions of the decoupled equations (15) and (17), and hence we may solve the latter system instead of the former.

Lemma 1. Let $\left(\phi_{m}, \vec{u}_{c}, p_{c}\right)$ be the solution of the coupled Navier-Stokes-Darcy system (11) and let $\left(\widehat{\phi}_{m}, \widehat{\vec{u}}_{c}, \widehat{p}_{c}\right)$ be the solution of the decoupled Navier-Stokes and Darcy equations (15) and (17) with Robin boundary conditions at the interface. Then, $\left(\widehat{\phi}_{m}, \widehat{\vec{u}}_{c}, \widehat{p}_{c}\right)=\left(\phi_{m}, \vec{u}_{c}, p_{c}\right)$ if and only if $\gamma_{f}, \gamma_{p}, \eta_{f}, \vec{\eta}_{f \tau}$, and $\eta_{p}$ satisfy the following compatibility conditions:

$$
\begin{gathered}
\eta_{p}=\gamma_{p} \widehat{\vec{u}}_{c} \cdot \vec{n}_{c}+g \widehat{\phi}_{m}, \\
\eta_{f}=\gamma_{f} \widehat{\vec{u}}_{c} \cdot \vec{n}_{c}-g \widehat{\phi}_{m}+g z .
\end{gathered}
$$




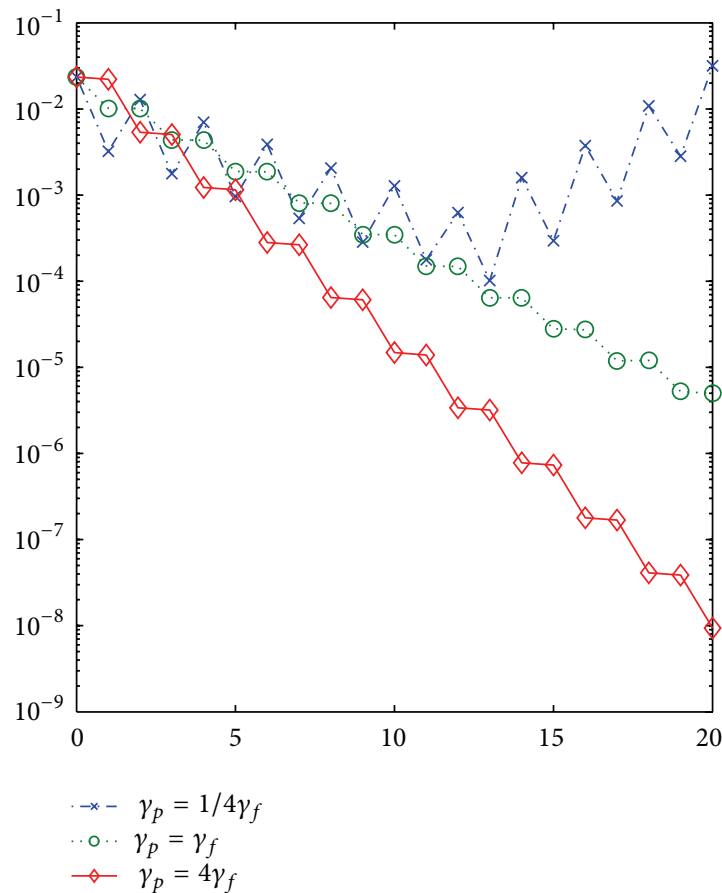

(a)

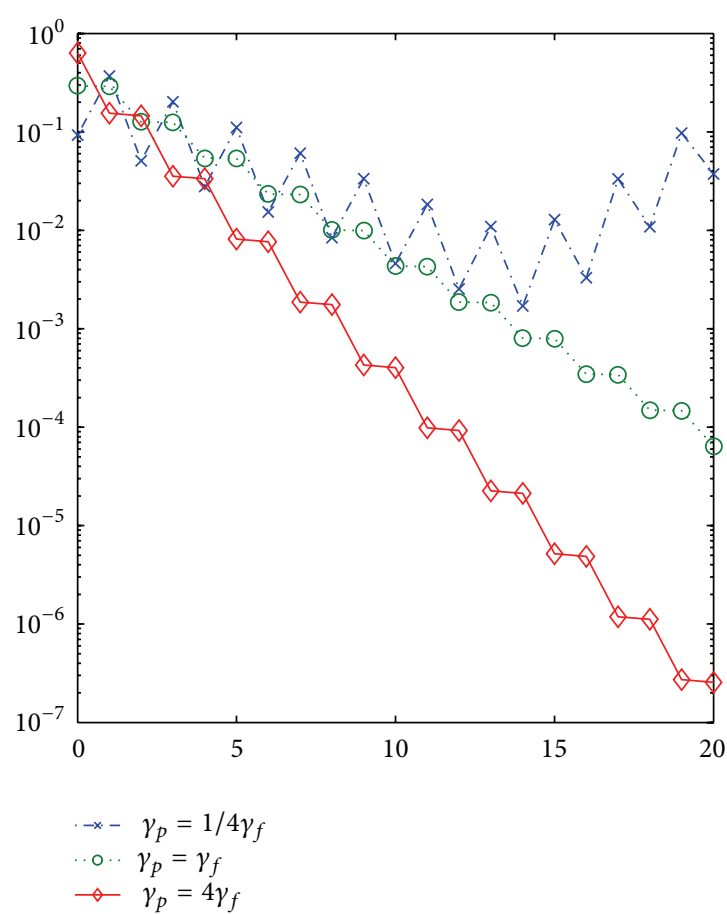

(b)

FIGURE 2: Convergence for the velocity of the free flow (a) and the hydraulic head of the porous medium flow (b) versus the iteration counter $m$ for the parallel DDM with BJS interface condition.

TABLE 1: Errors of the finite element method for the steady Navier-Stokes-Darcy model with BJS interface condition.

\begin{tabular}{lccccc}
\hline$h$ & $\left\|\vec{u}_{h}-\vec{u}\right\|_{0}$ & $\left\|\vec{u}_{h}-\vec{u}\right\|_{1}$ & $\left\|p_{h}-p\right\|_{0}$ & $\left\|\phi_{h}-\phi\right\|_{0}$ & $\left|\phi_{h}-\phi\right|_{1}$ \\
\hline $1 / 8$ & $1.367 \times 10^{-3}$ & $6.147 \times 10^{-2}$ & $8.002 \times 10^{-3}$ & $6.940 \times 10^{-4}$ & $2.452 \times 10^{-2}$ \\
$1 / 16$ & $1.687 \times 10^{-4}$ & $1.577 \times 10^{-2}$ & $8.559 \times 10^{-4}$ & $8.687 \times 10^{-5}$ & $6.187 \times 10^{-3}$ \\
$1 / 32$ & $2.086 \times 10^{-5}$ & $3.978 \times 10^{-3}$ & $9.506 \times 10^{-5}$ & $1.089 \times 10^{-5}$ & $1.553 \times 10^{-3}$ \\
$1 / 64$ & $2.594 \times 10^{-6}$ & $9.974 \times 10^{-4}$ & $1.121 \times 10^{-5}$ & $1.363 \times 10^{-6}$ & $3.890 \times 10^{-4}$ \\
$1 / 128$ & $3.235 \times 10^{-7}$ & $2.496 \times 10^{-4}$ & $1.363 \times 10^{-6}$ & $1.705 \times 10^{-7}$ & $9.733 \times 10^{-5}$ \\
\hline
\end{tabular}

Proof. Adding (15) and (17) together, we obtain the following: given $\eta_{p}, \eta_{f} \in L^{2}(\Gamma)$, find $\left(\widehat{\phi}_{m}, \widehat{u}_{f}, \widehat{p}_{c}\right) \in X_{\mathrm{m}} \times X_{c} \times Q_{c}$ such that

$$
\begin{aligned}
\left(\widehat{\vec{u}}_{c} \cdot \nabla \widehat{\vec{u}}_{c}, \vec{v}\right)_{\Omega_{c}}+a_{c}\left(\widehat{\vec{u}}_{c}, \vec{v}\right)+b_{c}\left(\vec{v}, \widehat{p}_{c}\right) \\
\quad-b_{c}\left(\widehat{\vec{u}}_{c}, q\right)+a_{m}\left(\widehat{\phi}_{m}, \psi\right)+\gamma_{f}\left\langle\widehat{\vec{u}}_{c} \cdot \vec{n}_{c}, \vec{v} \cdot \vec{n}_{c}\right\rangle \\
\quad+\left\langle\frac{g \widehat{\phi}_{m}}{\gamma_{p}}, \psi\right\rangle+\frac{\alpha \nu \sqrt{\mathbf{d}}}{\sqrt{\operatorname{trace}\left(\prod\right)}}\left\langle P_{\tau} \widehat{\vec{u}}_{c}, P_{\tau} \vec{v}\right\rangle \\
=\left(f_{m}, \psi\right)_{\Omega_{m}}+\left(\vec{f}_{c}, \vec{v}\right)_{\Omega_{c}}+\left\langle\eta_{f}, \vec{v} \cdot \vec{n}_{c}\right\rangle \\
\quad+\left\langle\frac{\eta_{p}}{\gamma_{p}}, \psi\right\rangle, \quad \forall(\vec{v}, q, \psi) \in X_{m} \times X_{c} \times Q_{c} .
\end{aligned}
$$

For the necessity of the lemma, we pick $\psi=0$ and $\vec{v}$ such that $P_{\tau} \vec{v}=0$ in (11) and (20); then by subtracting (20) from (11), we get

$$
\begin{array}{r}
\left\langle\eta_{f}-\gamma_{f} \vec{v}_{f} \cdot \vec{n}_{c}+g \phi_{m}-g z, \vec{v} \cdot \vec{n}_{c}\right\rangle=0, \\
\forall \vec{v} \in X_{c} \text { with } P_{\tau} \vec{v}=0,
\end{array}
$$

which implies (19). The necessity of (18) can be derived in a similar fashion.

As for the sufficiency of the lemma, by substituting the compatibility conditions (18)-(19), we easily see that $\left(\widehat{\phi}_{m}, \widehat{\vec{u}}_{c}, \widehat{p}_{c}\right)$ solves the coupled Navier-Stokes-Darcy system (11), which completes the proof.

Now we use the decoupled weak formulation constructed above to propose a physics-based parallel domain decomposition method with Newton iteration as follows. 


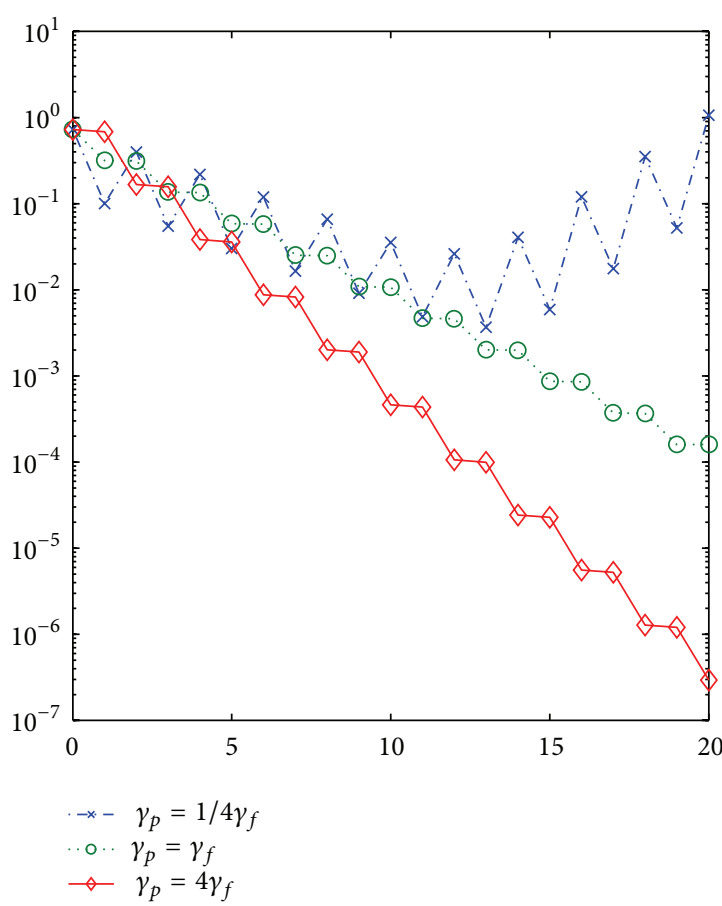

(a)

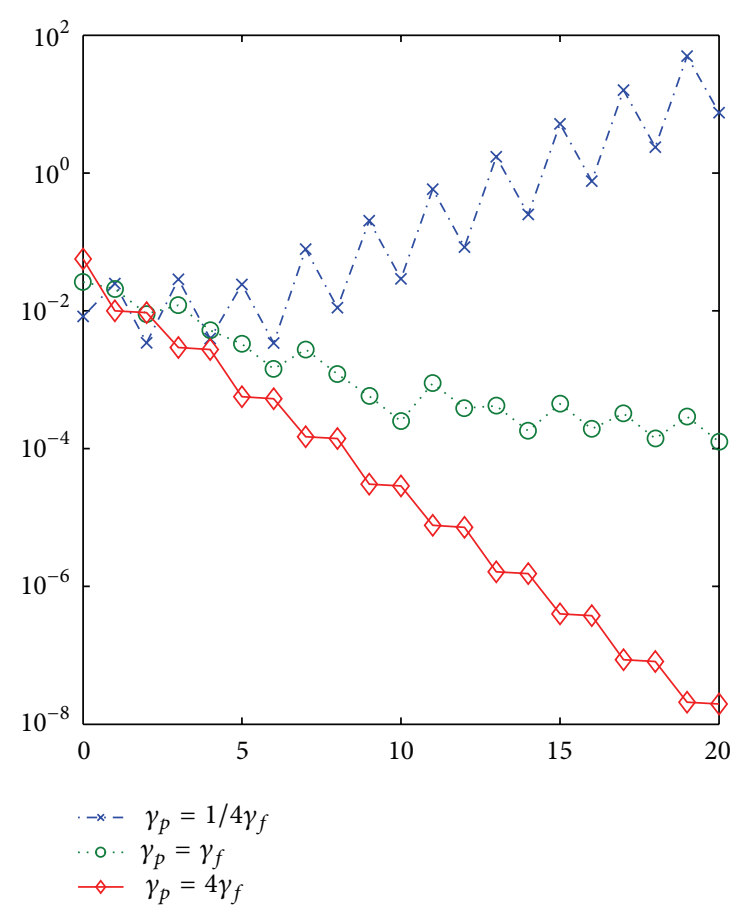

(b)

FIGURE 3: Convergence for the pressure of the free flow (a) and $\eta_{f}(\mathrm{~b})$ versus the iteration counter $m$ for the parallel DDM with BJS interface condition.

(1) Initial values $\eta_{p}^{0}$ and $\eta_{f}^{0}$ are guessed. They may be taken to be zero.

(2) For $k=0,1,2, \ldots$, independently solve the Darcy and Navier-Stokes equations constructed above. More precisely, $\phi_{m}^{k} \in X_{m}$ is computed from

$$
\begin{array}{r}
a_{m}\left(\phi_{m}^{k}, \psi\right)+\left\langle\frac{g \phi_{m}^{k}}{\gamma_{p}}, \psi\right\rangle=\left\langle\frac{\eta_{p}^{k}}{\gamma_{p}}, \psi\right\rangle+\left(f_{m}, \psi\right)_{\Omega_{m}} \\
\forall \psi \in X_{m}
\end{array}
$$

and $\vec{u}_{c}^{k} \in X_{c}$ and $p_{c}^{k} \in Q_{c}$ are computed from the following Newton iteration.

(i) Initial value $\vec{u}_{c}^{k, 0}$ is chosen for the Newton iteration. For instance, it may be taken to be $\vec{u}_{c}^{0,0}=0$ and $\vec{u}_{c}^{k, 0}=\vec{u}_{c}^{k-1}$ for $k=1,2, \ldots$.

(ii) For $m=0,1,2, \ldots, M$, solve

$$
\begin{aligned}
& \left(\vec{u}_{c}^{k, m+1} \cdot \nabla \vec{u}_{c}^{k, m}, \vec{v}\right)_{\Omega_{c}}+\left(\vec{u}_{c}^{k, m} \cdot \nabla \vec{u}_{c}^{k, m+1}, \vec{v}\right)_{\Omega_{c}} \\
& \quad+a_{c}\left(\vec{u}_{c}^{k, m+1}, \vec{v}\right)+b_{c}\left(\vec{v}, p_{c}^{k, m+1}\right) \\
& \quad-b_{c}\left(\vec{u}_{c}^{k, m+1}, q\right)+\gamma_{f}\left\langle\vec{u}_{c}^{k, m+1} \cdot \vec{n}_{c}, \vec{v} \cdot \vec{n}_{c}\right\rangle \\
& +\frac{\alpha \nu \sqrt{\mathbf{d}}}{\sqrt{\operatorname{trace}\left(\prod\right)}}\left\langle P_{\tau} \vec{u}_{c}^{k, m+1}, P_{\tau} \vec{v}\right\rangle
\end{aligned}
$$

$$
\begin{array}{r}
=\left(\vec{u}_{c}^{k, m} \cdot \nabla \vec{u}_{c}^{k, m}, \vec{v}\right)_{\Omega_{c}}+\left\langle\eta_{f}^{k}, \vec{v} \cdot \vec{n}_{c}\right\rangle+\left(\vec{f}_{c}, \vec{v}\right)_{\Omega_{c}}, \\
\forall(\vec{v}, q, \psi) \in X_{c} \times Q_{c} \times X_{m} .
\end{array}
$$

(iii) Set $\vec{u}_{c}^{k}=\vec{u}_{c}^{k, M+1}$ and $p_{c}^{k}=p_{c}^{k, M+1}$.

(3) $\eta_{p}^{k+1}$ and $\eta_{f}^{k+1}$ are updated in the following manner:

$$
\begin{aligned}
& \eta_{f}^{k+1}=\frac{\gamma_{f}}{\gamma_{p}} \eta_{p}^{k}-\left(1+\frac{\gamma_{f}}{\gamma_{p}}\right) g \phi_{m}^{k}+g z, \\
& \eta_{p}^{k+1}=-\eta_{f}^{k}+\left(\gamma_{f}+\gamma_{p}\right) \vec{u}_{c}^{k} \cdot \vec{n}_{c}+g z .
\end{aligned}
$$

Then the corresponding domain decomposition finite element method is proposed as follows.

(1) Initial values $\eta_{p, h}^{0}$ and $\eta_{f, h}^{0}$ are guessed. They may be taken to be zero.

(2) For $k=0,1,2, \ldots$, independently solve the Darcy and Navier-Stokes equations with the Robin boundary conditions on the interface, which are constructed previously. More precisely, $\phi_{m, h}^{k} \in X_{m h}$ is computed from

$$
\begin{aligned}
a_{m}\left(\phi_{m, h}^{k}, \psi_{h}\right)+\left\langle\frac{g \phi_{m, h}^{k}}{\gamma_{p}}, \psi_{h}\right\rangle & \\
& =\left\langle\frac{\eta_{p, h}^{k}}{\gamma_{p}}, \psi_{h}\right\rangle+\left(f_{m}, \psi_{h}\right)_{\Omega_{m}}, \quad \forall \psi_{h} \in X_{m h},
\end{aligned}
$$




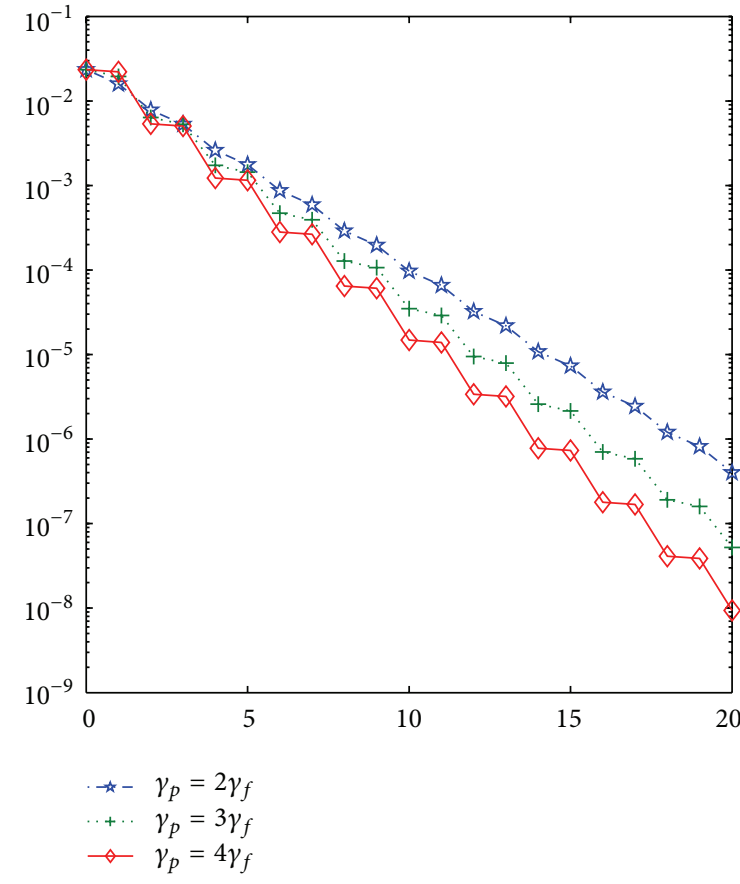

(a)

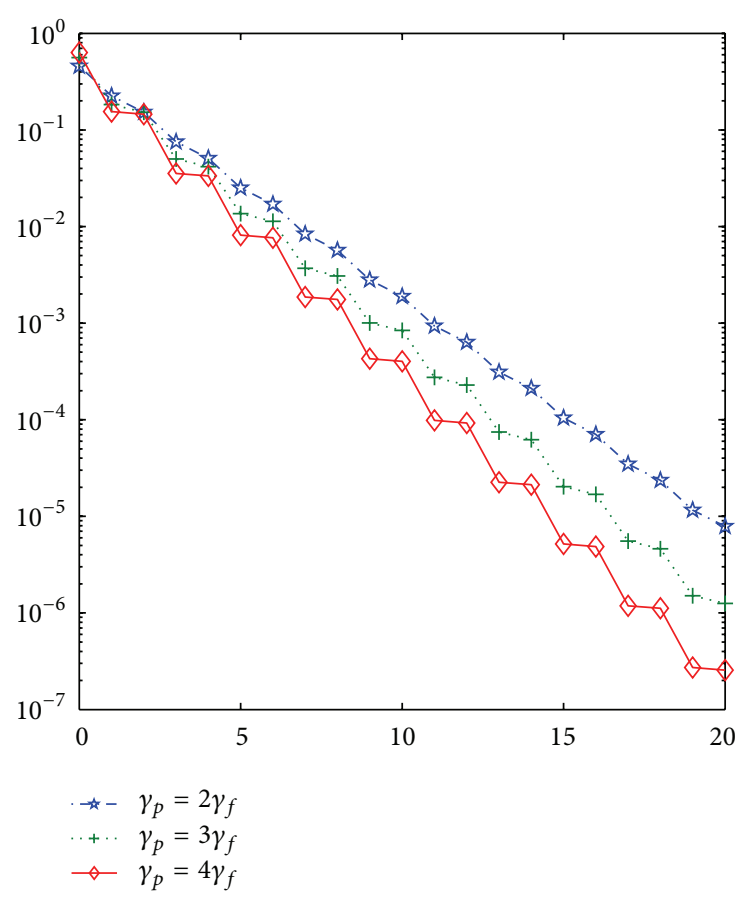

(b)

FIGURE 4: Geometric convergence rate of the velocity of the free flow (a) and the hydraulic head of the porous medium flow (b) for the parallel DDM with BJS interface condition.

and $\vec{u}_{c, h}^{k} \in X_{c h}$ and $p_{c, h}^{k} \in Q_{c h}$ are computed from the following Newton iteration.

(i) Initial value $\vec{u}_{c, h}^{k, 0}$ is chosen for the Newton iteration. For instance, it may be taken to be $\vec{u}_{c, h}^{0,0}=0$ and $\vec{u}_{c, h}^{k, 0}=\vec{u}_{c, h}^{k-1}$ for $k=1,2, \ldots$

(ii) For $m=0,1,2, \ldots, M$, solve

$$
\begin{aligned}
& \left(\vec{u}_{c, h}^{k, m+1} \cdot \nabla \vec{u}_{c, h}^{k, m}, \vec{v}_{h}\right)_{\Omega_{c}}+\left(\vec{u}_{c, h}^{k, m} \cdot \nabla \vec{u}_{c, h}^{k, m+1}, \vec{v}_{h}\right)_{\Omega_{c}} \\
& +a_{c}\left(\vec{u}_{c, h}^{k, m+1}, \vec{v}_{h}\right)+b_{c}\left(\vec{v}_{h}, p_{c}^{k, m+1}\right)-b_{c}\left(\vec{u}_{c, h}^{k, m+1}, q_{h}\right) \\
& +\gamma_{f}\left\langle\vec{u}_{c, h}^{k, m+1} \cdot \vec{n}_{c}, \vec{v}_{h} \cdot \vec{n}_{c}\right\rangle \\
& +\frac{\alpha \nu \sqrt{\mathbf{d}}}{\sqrt{\operatorname{trace}\left(\prod\right)}}\left\langle P_{\tau} \vec{u}_{c, h}^{k, m+1}, P_{\tau} \vec{v}_{h}\right\rangle \\
& =\left(\vec{u}_{c, h}^{k, m} \cdot \nabla \vec{u}_{c, h}^{k, m}, \vec{v}_{h}\right)_{\Omega_{c}}+\left\langle\eta_{f, h}^{k}, \vec{v}_{h} \cdot \vec{n}_{c}\right\rangle+\left(\vec{f}_{c}, \vec{v}_{h}\right)_{\Omega_{c}} \\
& \forall\left(\vec{v}_{h}, q_{h}, \psi_{h}\right) \in X_{c h} \times Q_{c h} \times X_{m h} .
\end{aligned}
$$

(iii) Set $\vec{u}_{c, h}^{k}=\vec{u}_{c, h}^{k, m+1}$ and $p_{c, h}^{k}=p_{c, h}^{k, M+1}$.

(3) $\eta_{p, h}^{k+1}$ and $\eta_{f, h}^{k+1}$ are updated in the following manner:

$$
\begin{aligned}
& \eta_{f, h}^{k+1}=\frac{\gamma_{f}}{\gamma_{p}} \eta_{p, h}^{k}-\left(1+\frac{\gamma_{f}}{\gamma_{p}}\right) g \phi_{m, h}^{k}+g z, \\
& \eta_{p, h}^{k+1}=-\eta_{f, h}^{k}+\left(\gamma_{f}+\gamma_{p}\right) \vec{u}_{c, h}^{k} \cdot \vec{n}_{c}+g z .
\end{aligned}
$$

\section{Numerical Example}

Example 1. Consider the model problem (2)-(6) with the BJS interface condition (7) on $\Omega=[0, \pi] \times[-1,1]$ with $\Omega_{m}=[0, \pi] \times[0,1]$ and $\Omega_{c}=[0, \pi] \times[-1,0]$. Choose

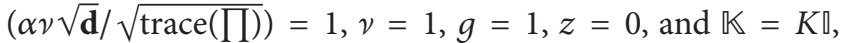

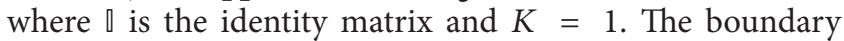
condition data functions and the source terms are chosen such that the exact solution is given by

$$
\begin{aligned}
\phi_{m}= & \left(e^{y}-e^{-y}\right) \sin (x) e^{t}, \\
\vec{u}_{c}= & {\left[\frac{K}{\pi} \sin (2 \pi y) \cos (x) e^{t},\right.} \\
& \left.\left(-2 K+\frac{K}{\pi^{2}} \sin ^{2}(\pi y)\right) \sin (x) e^{t}\right]^{T}, \\
p_{c}= & 0 .
\end{aligned}
$$

We divide $\Omega_{m}$ and $\Omega_{c}$ into rectangles of height $h=1 / N$ and width $\pi h$, where $N$ is a positive integer, and then subdivide each rectangle into two triangles by drawing a diagonal. The Taylor-Hood element pair is used for the Navier-Stokes equations, and the quadratic finite element is used for the second-order formulation of the Darcy equation.

For the coupled finite element method of the steady Navier-Stokes-Darcy model with BJS interface condition, 


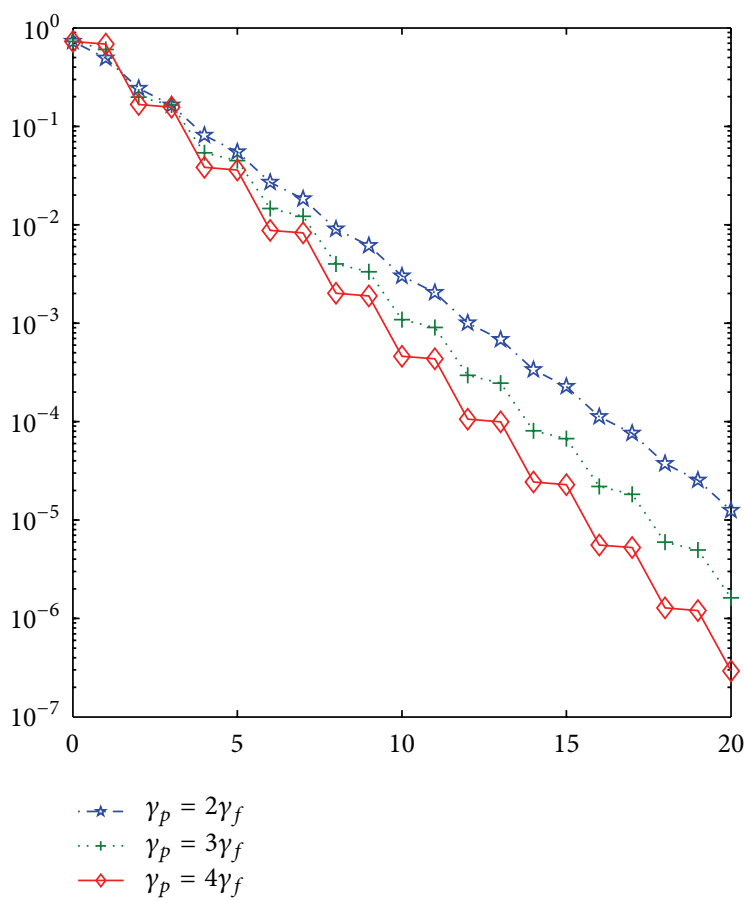

(a)

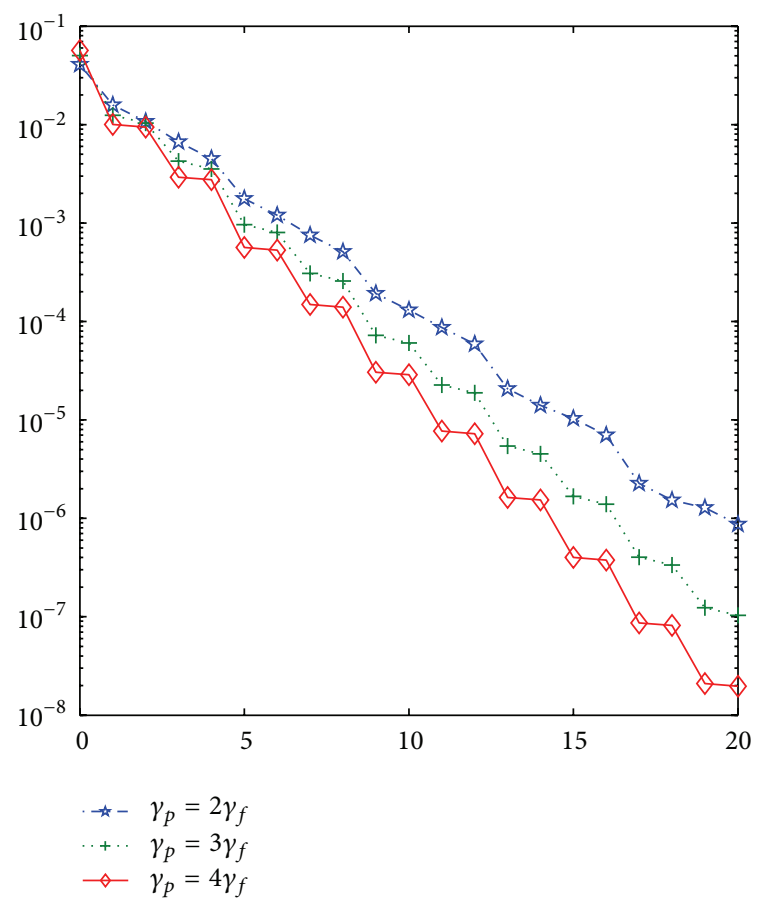

(b)

FIGURE 5: Geometric convergence rate of the pressure of the free flow (a) and $\eta_{f}$ (b) versus the iteration counter $m$ for the parallel DDM with BJS interface condition.

TABLE 2: $L^{2}$ errors in velocity and hydraulic head for the parallel DDM with BJS interface condition.

\begin{tabular}{|c|c|c|c|c|}
\hline & $L^{2}$ velocity errors & $e(i) / e(i-4)$ & $L^{2}$ hydraulic head errors & $e(i) / e(i-4)$ \\
\hline$e(0)$ & $2.342 \times 10^{-2}$ & & $6.338 \times 10^{-1}$ & \\
\hline$e(4)(i=4)$ & $1.225 \times 10^{-3}$ & 0.0523 & $3.337 \times 10^{-2}$ & 0.0527 \\
\hline$e(8)(i=8)$ & $6.450 \times 10^{-5}$ & 0.0527 & $1.756 \times 10^{-3}$ & 0.0526 \\
\hline$e(12)(i=12)$ & $3.395 \times 10^{-6}$ & 0.0526 & $9.246 \times 10^{-5}$ & 0.0527 \\
\hline$e(16)(i=16)$ & $1.787 \times 10^{-7}$ & 0.0526 & $4.868 \times 10^{-6}$ & 0.0527 \\
\hline$e(20)(i=20)$ & $9.409 \times 10^{-9}$ & 0.0527 & $2.562 \times 10^{-7}$ & 0.0526 \\
\hline
\end{tabular}

Table 1 provides errors for different choices of $h$. Using linear regression, the errors in Table 1 satisfy

$$
\begin{aligned}
& \left\|\vec{u}_{c, h}-\vec{u}_{c}\right\|_{0} \approx 0.714 h^{3.011}, \quad\left|\vec{u}_{c, h}-\vec{u}_{c}\right|_{1} \approx 3.867 h^{1.987}, \\
& \left\|p_{c, h}-p_{c}\right\|_{0} \approx 5.123 h^{3.129} \text {, } \\
& \left\|\phi_{m, h}-\phi_{m}\right\|_{0} \approx 0.354 h^{2.998}, \quad\left|\phi_{m, h}-\phi_{m}\right|_{1} \approx 1.556 h^{1.995} \text {. }
\end{aligned}
$$

These rates of convergence are consistent with the approximation capability of the Taylor-Hood element and quadratic element, which is third order with respect to $L^{2}$ norm of $\vec{u}_{c}$ and $\phi_{m}$, second order with respect to $H^{1}$ norm of $\vec{u}_{c}$ and $\phi_{m}$, and second order with respect to $L^{2}$ norms of $p_{c}$. In particular, the third-order convergence rate of $p_{c}$ observed above, which is better than the approximation capability of the linear element, is mainly due to the special analytic solution $p=0$.
For the parallel DDM with $v=1, K=1, \gamma_{f}=0.3$, and $h=1 / 32$, Figures 2 and 3 show the $L^{2}$ errors of hydraulic head, velocity, pressure, and $\eta_{f}$. We can see that the parallel domain decomposition method is convergent for $\gamma_{f} \leq \gamma_{p}$. Moreover, Figures 4 and 5 show that a smaller $\gamma_{f} / \gamma_{p}$ leads to faster convergence.

Then Tables 2 and 3 list some $L^{2}$ errors in velocity, hydraulic head, pressure, and $\eta_{f}$ for the parallel domain decomposition method with $\gamma_{f}=0.3$ and $\gamma_{p}=1.2$. The data in these two tables indicate the geometric convergence rate $\sqrt{\gamma_{f} / \gamma_{p}}$ since all the error ratios are less than $\left(\sqrt{\gamma_{f} / \gamma_{p}}\right)^{4}=$ $(\sqrt{1 / 4})^{4}=0.0625$.

Finally, for the preconditioning feature of the domain decomposition method, Table 4 shows the number of iterations $M$ is independent of the grid size $h$. Here, we set $\gamma_{S}=$ $0.3, \gamma_{D}=1.2, v=1$, and $K=1$. Let $\phi_{h}^{k}, \vec{u}_{h}^{k}$, and $p_{h}^{k}$ denote the finite element solutions of $\phi_{D}^{k}, \vec{u}_{S}^{k}$, and $p_{S}^{k}$ at the $k$ th step 
TABLE 3: $L^{2}$ errors in pressure and $\eta_{f}$ for the parallel DDM with BJS interface condition.

\begin{tabular}{|c|c|c|c|c|}
\hline & $L^{2}$ velocity errors & $e(i) / e(i-4)$ & $L^{2}$ hydraulic head errors & $e(i) / e(i-4)$ \\
\hline$e(0)$ & $7.268 \times 10^{-1}$ & & $5.668 \times 10^{-2}$ & \\
\hline$e(4)(i=4)$ & $3.826 \times 10^{-2}$ & 0.0526 & $2.752 \times 10^{-3}$ & 0.0486 \\
\hline$e(8)(i=8)$ & $2.014 \times 10^{-3}$ & 0.0526 & $1.399 \times 10^{-4}$ & 0.0508 \\
\hline$e(12)(i=12)$ & $1.060 \times 10^{-4}$ & 0.0526 & $7.233 \times 10^{-6}$ & 0.0517 \\
\hline$e(16)(i=16)$ & $5.579 \times 10^{-6}$ & 0.0526 & $3.767 \times 10^{-7}$ & 0.0521 \\
\hline$e(20)(i=20)$ & $2.937 \times 10^{-7}$ & 0.0526 & $1.969 \times 10^{-8}$ & 0.0523 \\
\hline
\end{tabular}

TABLE 4: The iteration counter $M$ versus the grid size $h$ for both the parallel Robin-Robin domain decomposition method with BJS interface condition.

\begin{tabular}{lcccc}
\hline$h$ & $1 / 8$ & $1 / 16$ & $1 / 32$ & $1 / 64$ \\
$M$ & 19 & 19 & 19 & 19 \\
\hline
\end{tabular}

of the domain decomposition algorithm. The criterion used to stop the iteration, that is, to determine the value $M$, is $\left\|\vec{u}_{h}^{k}-\vec{u}_{h}^{k-1}\right\|_{0}+\left\|\phi_{h}^{k}-\phi_{h}^{k-1}\right\|_{0}+\left\|p_{h}^{k}-p_{h}^{k-1}\right\|_{0}<\varepsilon$, where the tolerance $\varepsilon=10^{-5}$.

\section{Conclusions}

In this paper, a parallel physics-based domain decomposition method is proposed for the stationary Navier-Stokes-Darcy model with the BJS interface condition. This method is based on the Robin boundary conditions constructed from the three physical interface conditions. Moreover, it is convergent with geometric convergence rates if the relaxation parameter is selected properly. The number of iteration steps is independent of the grid size due to the natural preconditioning advantage of the domain decomposition methods.

\section{Acknowledgments}

This work is partially supported by DOE Grant DEFE0009843, National Natural Science Foundation of China (11175052).

\section{References}

[1] T. Arbogast and D. S. Brunson, "A computational method for approximating a Darcy-Stokes system governing a vuggy porous medium," Computational Geosciences, vol. 11, no. 3, pp. 207-218, 2007.

[2] T. Arbogast and M. S. M. Gomez, "A discretization and multigrid solver for a Darcy-Stokes system of three dimensional vuggy porous media," Computational Geosciences, vol. 13, no. 3, pp. 331-348, 2009.

[3] T. Arbogast and H. L. Lehr, "Homogenization of a DarcyStokes system modeling vuggy porous media," Computational Geosciences, vol. 10, no. 3, pp. 291-302, 2006.

[4] Y. Cao, M. Gunzburger, X. Hu, F. Hua, X. Wang, and W. Zhao, "Finite element approximations for Stokes-Darcy flow with Beavers-Joseph interface conditions," SIAM Journal on Numerical Analysis, vol. 47, no. 6, pp. 4239-4256, 2010.
[5] Y. Cao, M. Gunzburger, F. Hua, and X. Wang, "Coupled Stokes-Darcy model with Beavers-Joseph interface boundary condition," Communications in Mathematical Sciences, vol. 8, no. 1, pp. 1-25, 2010.

[6] M. Discacciati, Domain decomposition methods for the coupling of surface and groundwater flows [Ph.D. thesis], École Polytechnique Fédérale de Lausanne, Lausanne, Switzerland, 2004.

[7] V. J. Ervin, E. W. Jenkins, and S. Sun, "Coupled generalized nonlinear Stokes flow with flow through a porous medium," SIAM Journal on Numerical Analysis, vol. 47, no. 2, pp. 929-952, 2009.

[8] M. Moraiti, "On the quasistatic approximation in the StokesDarcy model of groundwater-surface water flows," Journal of Mathematical Analysis and Applications, vol. 394, no. 2, pp. 796808, 2012.

[9] I. Babuška and G. N. Gatica, "A residual-based a posteriori error estimator for the Stokes-Darcy coupled problem," SIAM Journal on Numerical Analysis, vol. 48, no. 2, pp. 498-523, 2010.

[10] Y. Cao, M. Gunzburger, X. He, and X. Wang, "Robin-Robin domain decomposition methods for the steady-state StokesDarcy system with the Beavers-Joseph interface condition," Numerische Mathematik, vol. 117, no. 4, pp. 601-629, 2011.

[11] Y. Cao, M. Gunzburger, X.-M. He, and X. Wang, "Parallel, non-iterative, multi-physics domain decomposition methods for time-dependent Stokes-Darcy systems," Mathematics of Computation. In press.

[12] W. Chen, M. Gunzburger, F. Hua, and X. Wang, "A parallel Robin-Robin domain decomposition method for the StokesDarcy system," SIAM Journal on Numerical Analysis, vol. 49, no. 3, pp. 1064-1084, 2011.

[13] M. Discacciati, "Iterative methods for Stokes/Darcy coupling," in Domain Decomposition Methods in Science and Engineering, vol. 40 of Lecture Notes in Computational Science and Engineering, pp. 563-570, Springer, Berlin, Germany, 2005.

[14] M. Discacciati, E. Miglio, and A. Quarteroni, "Mathematical and numerical models for coupling surface and groundwater flows," Applied Numerical Mathematics, vol. 43, no. 1-2, pp. 57-74, 2002, 19th Dundee Biennial Conference on Numerical Analysis (2001).

[15] M. Discacciati and A. Quarteroni, "Analysis of a domain decomposition method for the coupling of Stokes and Darcy equations," in Numerical Mathematics and Advanced Applications, pp. 3-20, Springer, Milan, Italy, 2003.

[16] M. Discacciati and A. Quarteroni, "Convergence analysis of a subdomain iterative method for the finite element approximation of the coupling of Stokes and Darcy equations," Computing and Visualization in Science, vol. 6, no. 2-3, pp. 93-103, 2004. 
[17] M. Discacciati, A. Quarteroni, and A. Valli, "Robin-Robin domain decomposition methods for the Stokes-Darcy coupling," SIAM Journal on Numerical Analysis, vol. 45, no. 3, pp. 1246-1268, 2007.

[18] W. Feng, X. He, Z. Wang, and X. Zhang, "Non-iterative domain decomposition methods for a non-stationary StokesDarcy model with Beavers-Joseph interface condition," Applied Mathematics and Computation, vol. 219, no. 2, pp. 453-463, 2012.

[19] G. N. Gatica, S. Meddahi, and R. Oyarzúa, "A conforming mixed finite-element method for the coupling of fluid flow with porous media flow," IMA Journal of Numerical Analysis, vol. 29, no. 1, pp. 86-108, 2009.

[20] G. N. Gatica, R. Oyarzúa, and F.-J. Sayas, "A residual-based a posteriori error estimator for a fully-mixed formulation of the Stokes-Darcy coupled problem," Computer Methods in Applied Mechanics and Engineering, vol. 200, no. 21-22, pp. 1877-1891, 2011.

[21] R. H. W. Hoppe, P. Porta, and Y. Vassilevski, "Computational issues related to iterative coupling of subsurface and channel flows," Calcolo, vol. 44, no. 1, pp. 1-20, 2007.

[22] B. Jiang, "A parallel domain decomposition method for coupling of surface and groundwater flows," Computer Methods in Applied Mechanics and Engineering, vol. 198, no. 9-12, pp. 947957, 2009.

[23] W. Layton, H. Tran, and X. Xiong, "Long time stability of four methods for splitting the evolutionary Stokes-Darcy problem into Stokes and Darcy subproblems," Journal of Computational and Applied Mathematics, vol. 236, no. 13, pp. 3198-3217, 2012.

[24] W. J. Layton, F. Schieweck, and I. Yotov, "Coupling fluid flow with porous media flow," SIAM Journal on Numerical Analysis, vol. 40, no. 6, pp. 2195-2218, 2002.

[25] L. Shan, H. Zheng, and W. J. Layton, "A decoupling method with different subdomain time steps for the nonstationary Stokes-Darcy model," Numerical Methods for Partial Differential Equations, vol. 29, no. 2, pp. 549-583, 2013.

[26] M. Mu and X. Zhu, "Decoupled schemes for a non-stationary mixed Stokes-Darcy model," Mathematics of Computation, vol. 79, no. 270, pp. 707-731, 2010.

[27] C. Bernardi, T. C. Rebollo, F. Hecht, and Z. Mghazli, "Mortar finite element discretization of a model coupling Darcy and Stokes equations," Mathematical Modelling and Numerical Analysis, vol. 42, no. 3, pp. 375-410, 2008.

[28] Y. Boubendir and S. Tlupova, "Stokes-Darcy boundary integral solutions using preconditioners," Journal of Computational Physics, vol. 228, no. 23, pp. 8627-8641, 2009.

[29] W. Chen, P. Chen, M. Gunzburger, and N. Yan, "Superconvergence analysis of FEMs for the Stokes-Darcy system," Mathematical Methods in the Applied Sciences, vol. 33, no. 13, pp. 1605-1617, 2010.

[30] C. D’Angelo and P. Zunino, "Robust numerical approximation of coupled Stokes' and Darcy's flows applied to vascular hemodynamics and biochemical transport," Mathematical Modelling and Numerical Analysis, vol. 45, no. 3, pp. 447-476, 2011.

[31] M.-F. Feng, R.-S. Qi, R. Zhu, and B.-T. Ju, "Stabilized CrouzeixRaviart element for the coupled Stokes and Darcy problem," Applied Mathematics and Mechanics, vol. 31, no. 3, pp. 393-404, 2010.

[32] J. Galvis and M. Sarkis, "Balancing domain decomposition methods for mortar coupling Stokes-Darcy systems," in Domain Decomposition Methods in Science and Engineering XVI, vol. 55 of Lecture Notes in Computational Science and Engineering, pp. 373-380, Springer, Berlin, Germany, 2007.

[33] J. Galvis and M. Sarkis, "Non-matching mortar discretization analysis for the coupling Stokes-Darcy equations," Electronic Transactions on Numerical Analysis, vol. 26, pp. 350-384, 2007.

[34] J. Galvis and M. Sarkis, "FETI and BDD preconditioners for Stokes-Mortar-Darcy systems," Communications in Applied Mathematics and Computational Science, vol. 5, pp. 1-30, 2010.

[35] G. N. Gatica, R. Oyarzúa, and F.-J. Sayas, "Convergence of a family of Galerkin discretizations for the Stokes-Darcy coupled problem," Numerical Methods for Partial Differential Equations. An International Journal, vol. 27, no. 3, pp. 721-748, 2011.

[36] G. Kanschat and B. Rivière, "A strongly conservative finite element method for the coupling of Stokes and Darcy flow," Journal of Computational Physics, vol. 229, no. 17, pp. 5933-5943, 2010.

[37] T. Karper, K.-A. Mardal, and R. Winther, "Unified finite element discretizations of coupled Darcy-Stokes flow," Numerical Methods for Partial Differential Equations, vol. 25, no. 2, pp. 311-326, 2009.

[38] S. Khabthani, L. Elasmi, and F. Feuillebois, "Perturbation solution of the coupled Stokes-Darcy problem," Discrete and Continuous Dynamical Systems B, vol. 15, no. 4, pp. 971-990, 2011.

[39] M. Mu and J. Xu, "A two-grid method of a mixed Stokes-Darcy model for coupling fluid flow with porous media flow," SIAM Journal on Numerical Analysis, vol. 45, no. 5, pp. 1801-1813, 2007.

[40] S. Münzenmaier and G. Starke, "First-order system least squares for coupled Stokes-Darcy flow," SIAM Journal on Numerical Analysis, vol. 49, no. 1, pp. 387-404, 2011.

[41] B. Rivière, "Analysis of a discontinuous finite element method for the coupled Stokes and Darcy problems," Journal of Scientific Computing, vol. 22-23, pp. 479-500, 2005.

[42] B. Rivière and I. Yotov, "Locally conservative coupling of Stokes and Darcy flows," SIAM Journal on Numerical Analysis, vol. 42, no. 5, pp. 1959-1977, 2005.

[43] H. Rui and R. Zhang, "A unified stabilized mixed finite element method for coupling Stokes and Darcy flows," Computer Methods in Applied Mechanics and Engineering, vol. 198, no. 33-36, pp. 2692-2699, 2009.

[44] S. Tlupova and R. Cortez, "Boundary integral solutions of coupled Stokes and Darcy flows," Journal of Computational Physics, vol. 228, no. 1, pp. 158-179, 2009.

[45] J. M. Urquiza, D. N’Dri, A. Garon, and M. C. Delfour, "Coupling Stokes and Darcy equations," Applied Numerical Mathematics, vol. 58, no. 5, pp. 525-538, 2008.

[46] L. Badea, M. Discacciati, and A. Quarteroni, "Numerical analysis of the Navier-Stokes/Darcy coupling," Numerische Mathematik, vol. 115, no. 2, pp. 195-227, 2010.

[47] M. Cai, M. Mu, and J. Xu, "Numerical solution to a mixed Navier-Stokes/Darcy model by the two-grid approach," SIAM Journal on Numerical Analysis, vol. 47, no. 5, pp. 3325-3338, 2009.

[48] A. Çeşmelioğlu and B. Rivière, "Analysis of time-dependent Navier-Stokes flow coupled with Darcy flow," Journal of Numerical Mathematics, vol. 16, no. 4, pp. 249-280, 2008.

[49] A. Çeşmelioğlu and B. Rivière, "Primal discontinuous Galerkin methods for time-dependent coupled surface and subsurface flow," Journal of Scientific Computing, vol. 40, no. 1-3, pp. 115140, 2009. 
[50] P. Chidyagwai and B. Rivière, "On the solution of the coupled Navier-Stokes and Darcy equations," Computer Methods in Applied Mechanics and Engineering, vol. 198, no. 47-48, pp. 3806-3820, 2009.

[51] V. Girault and B. Rivière, "DG approximation of coupled Navier-Stokes and Darcy equations by Beaver-Joseph-Saffman interface condition," SIAM Journal on Numerical Analysis, vol. 47, no. 3, pp. 2052-2089, 2009.

[52] G. Beavers and D. Joseph, "Boundary conditions at a naturally permeable wall," Journal of Fluid Mechanics, vol. 30, pp. 197-207, 1967.

[53] W. Jäger and A. Mikelić, "On the interface boundary condition of Beavers, Joseph, and Saffman," SIAM Journal on Applied Mathematics, vol. 60, no. 4, pp. 1111-1127, 2000.

[54] I. P. Jones, "Low Reynolds number flow past a porous spherical shell," Proceedings of the Cambridge Philosophical Society, vol. 73, pp. 231-238, 1973.

[55] P. Saffman, "On the boundary condition at the interface of a porous medium," Studies in Applied Mathematics, vol. 1, pp. 7784, 1971.

[56] V. Girault and P.-A. Raviart, Finite Element Methods for NavierStokes Equations. Theory and Algorithms, vol. 5 of Springer Series in Computational Mathematics, Springer, Berlin, Germany, 1986.

[57] M. D. Gunzburger, Finite Element Methods for Viscous Incompressible Flows. A Guide to Theory, Practice, and Algorithms, Computer Science and Scientific Computing, Academic Press, Boston, Mass, USA, 1989. 


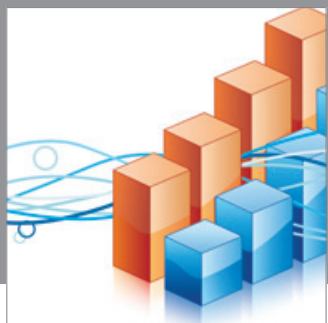

Advances in

Operations Research

mansans

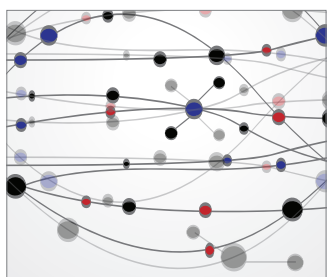

The Scientific World Journal
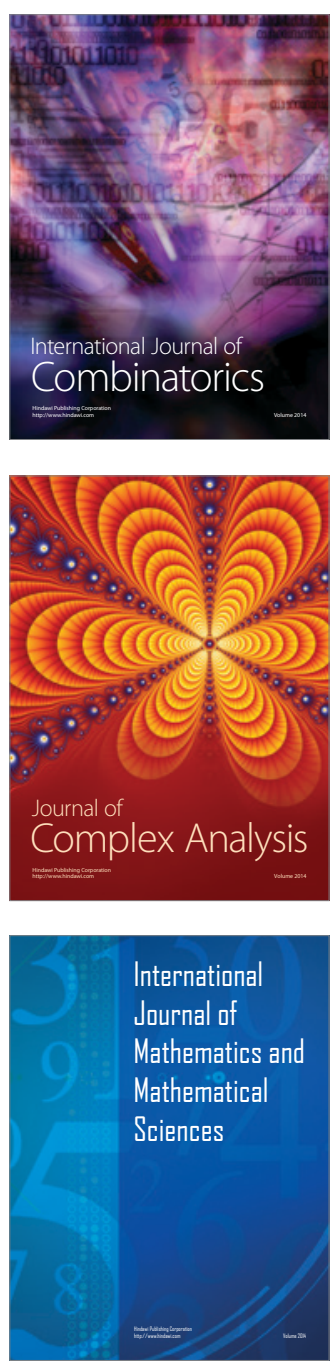
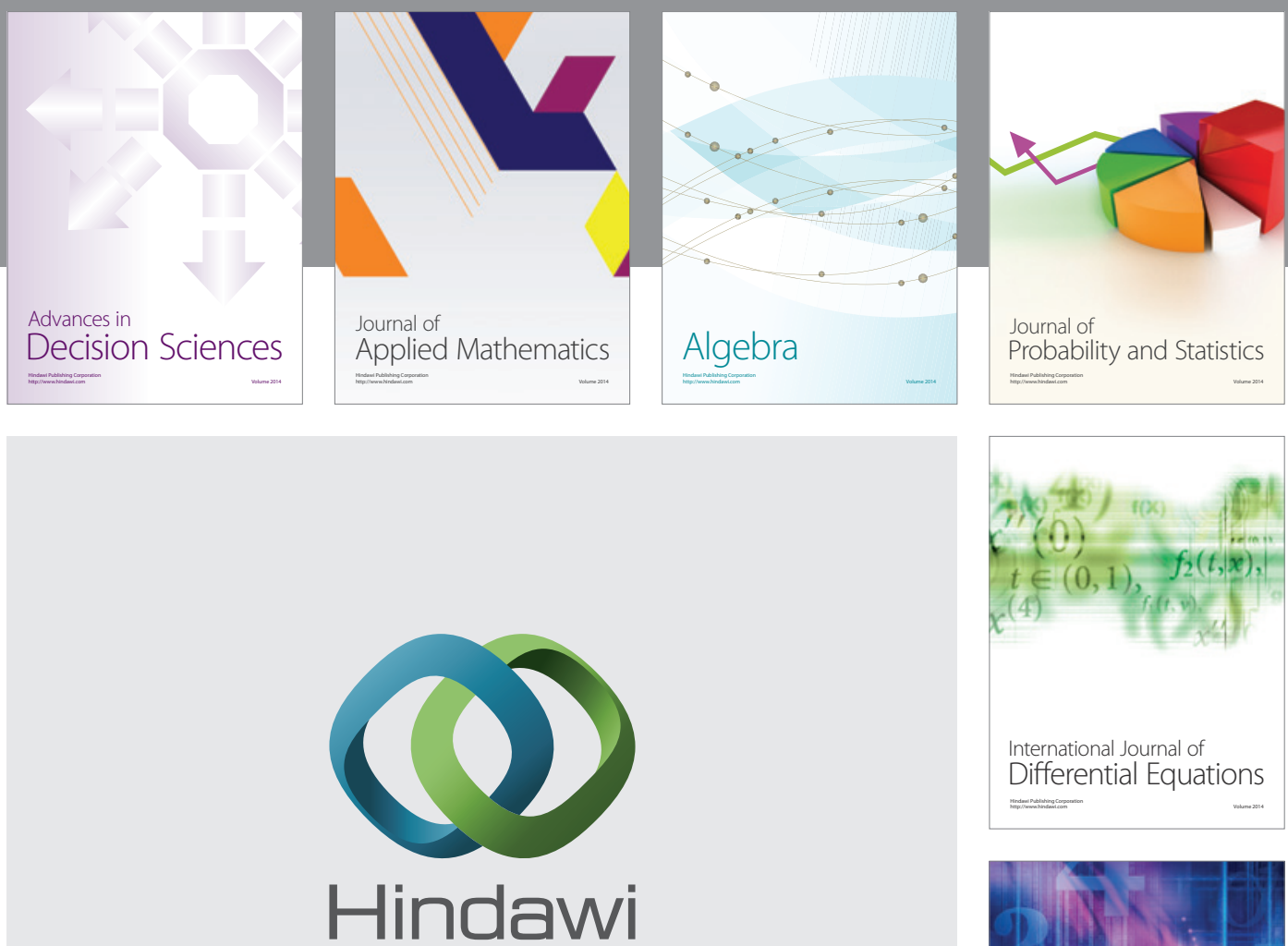

Submit your manuscripts at http://www.hindawi.com
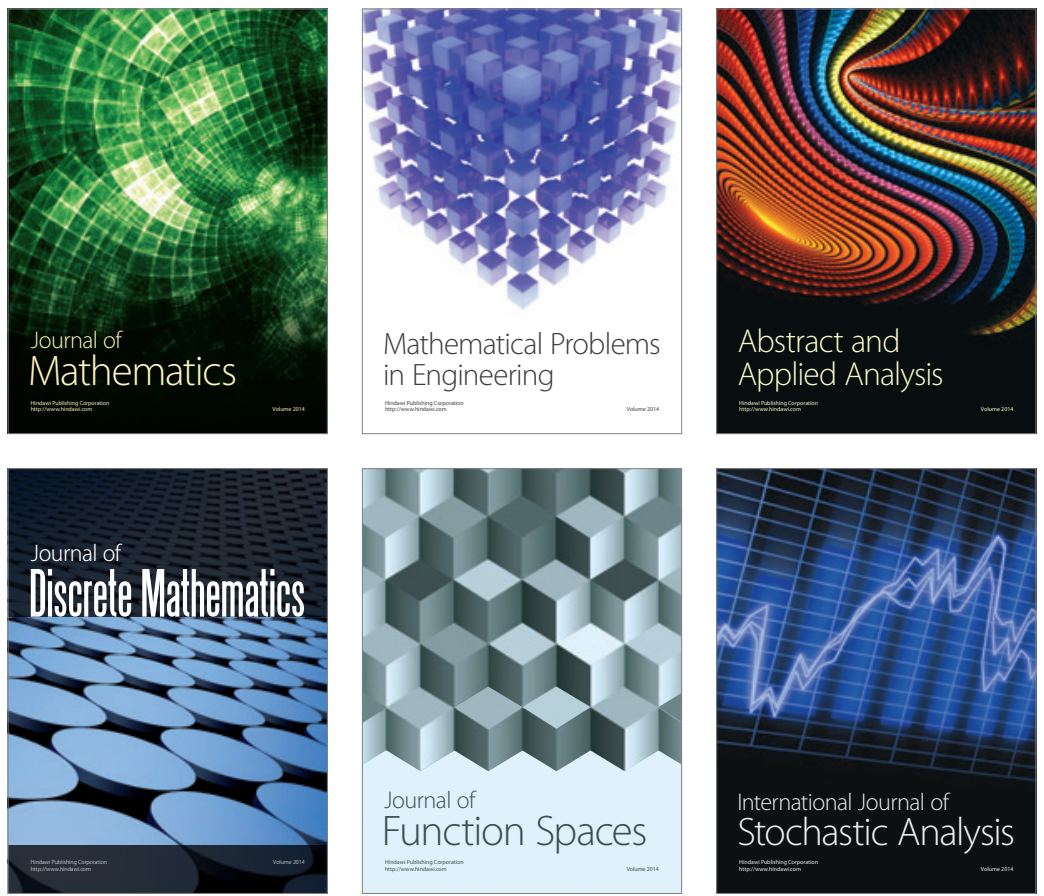

Journal of

Function Spaces

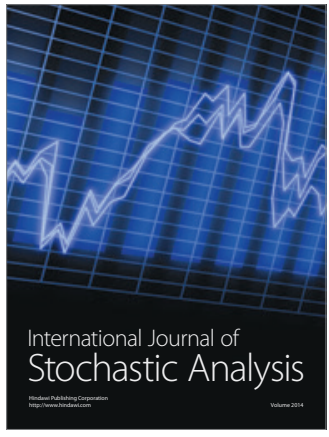

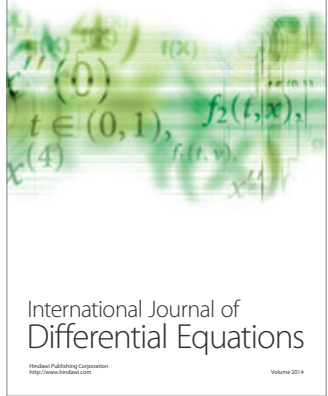
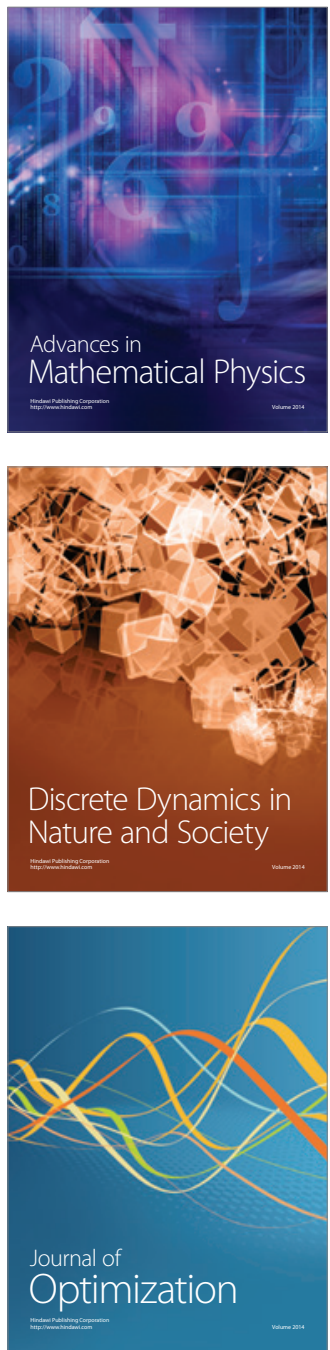